REGULAR PAPER

\title{
Determination of Li Abundances in Chondrules, Bulk Chondrites, and Standard Rocks by Direct-Loading Isotope Dilution Mass Spectrometry
}

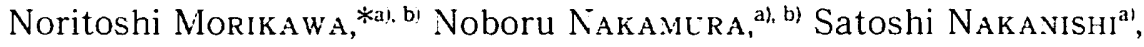 \\ Koshi Yamamoto, ${ }^{\text {(i), ci }}$ and Yurie TANAmI ${ }^{\text {al }}$
}

(Reccived August 16, 1995)

\begin{abstract}
Precise isotope dilution technique of $\mathrm{Li}$, along with ot her alkalis, alkaline earths and iron, for tiny silicate materials (having as low as $10 \mathrm{pg}$ Li) is described. Samples were decomposed by acids, equilibrated with mixed spikes including ${ }^{6} \mathrm{Li}$ and directly loaded on a mass spectrometer filament without chemical separation. $\mathrm{Li}$ and other lithophiles were measured by successive heating procedures. Results of precise Li analyses are presented for 7 Allende chondrules, 5 bulk chondrites and three standard rocks, JB-1, JB-2, and BCR-1.
\end{abstract}

\section{Introduction}

Lithium along with Be and B has an anomalously low cosmic abundance as a light element and has been argued for its origin in stellar environments. " Bulk analyses of $\mathrm{Li}$ have been systematically carried out for meteorites in two to three decade in several laboratories. ${ }^{2-51}$ However, except for a few cases, $^{\text {ij-i) }}$ individual meteoritic components such as chondrules have rarely been analyzed.

Lithium was reported to show abundance features similar to those for $\mathrm{Mg}$ for bulk meteorites, ${ }^{8)}$ but has not been sufficiently clarified for its cosmochemical properties. Although neutron activation analysis has been applied to determination of $\mathrm{Li}$ in meteorites, ${ }^{5}$ its application to a trace analysis of $\mathrm{Li}$ for meteoritic components such as chondrules ( $\mathrm{Li} \sim 2 \mathrm{ppm}$ ) have been limited. In view of current discoveries of heterogeneous distributions of elements and isotopes in primitive meteorites, it is important to clarify micro-distributions of trace elements and thus analysis of trace $\mathrm{Li}$ is considered to be of potential importance. In this connection, ion probe can be applicable. However, it is still of limited use in "bulk" elemental analyses for somewhat "larger" samples.

Previously, we have developed direct-loading isotope dilution mass spectrometry (DL-IDMS) technique of rare earth elements and other lithophiles for microgram-sized meteoritic materials. ${ }^{9 !}$ Extending our previous works, we have established the DL-IDMS

a) Department of Earth and Planetary Sciences, Faculty of Science, Kobe University (Nada, Kobe 657, Japan)

b Division of Environmental Science, Graduate School of Science and Technology, Kobe University (Nada. Kobe 65\%, Japan)

'Present address: Department of Earth and Planetary Sciences, School of Science, Nagoya University (Chikusa. Nagoya 464-01. Japan) 
technique of $\mathrm{Li}$ and applied it to individual chondrules, bulk chondrites and standard rocks in this work.

\section{Experimental}

A ${ }^{6} \mathrm{Li}$ enriched metal ball (purity: 99.954\%) immersed in kelocine medium was obtained from Oak Ridge National Laboratory (ORNL). The ${ }^{6} \mathrm{Li}$ metal was carefully cleaned by removing surface materials completely using a blade cutter and dissolved in distilled water $(\mathrm{Li} \sim 3500 \mathrm{ppm})$. A diluted ${ }^{6} \mathrm{Li}$-enriched spike solution was prepared by adjusting acidity ( $1 \mathrm{~N}-\mathrm{HCl}$ ) and concentrations ( $\mathrm{Li} \sim 7 \mathrm{ppm}$ ) and stored in a teflon bottle. For isotope dilution analyses, composite spike solutions including enriched isotopes of ${ }^{6} \mathrm{Li},{ }^{41} \mathrm{~K},{ }^{87} \mathrm{Rb},{ }^{84} \mathrm{Sr}$, and ${ }^{136} \mathrm{Ba}$ were prepared. Spike solutions of ${ }^{25} \mathrm{Mg}$, ${ }^{42} \mathrm{Ca}$, and ${ }^{56} \mathrm{Fe}$ had been prepared separately."

For isotopic reference, three laboratory standard solutions $\left(\mathrm{LiCl} \cdot \mathrm{H}_{2} \mathrm{O}\right.$ reagent [Merck; purity 99.99\%], the Allende bulk meteorite and Allende barred-olivine chondrule) were prepared. Because of hygroscopic properties of $\mathrm{LiCl} \cdot \mathrm{H}_{2} \mathrm{O}$, much cautions were paid for weighing procedure. For determination of $\mathrm{Li}$ concentration in ${ }^{6} \mathrm{Li}$ enriched spike solution, three mixed solutions having different relative amounts of $\mathrm{Li}$ reagent standard and ${ }^{6} \mathrm{Li}$-enriched spike solutions were prepared and six separate runs were carried out for the mixed solutions.

Sample decomposition procedures and mass spectrometric runs (using JEOL JMS05RB mass spectrometer) are similar to those previously described.9. For the standard rocks, about $10 \sim 50 \mu \mathrm{g}$-sized samples were directly loaded on filaments. On the other hand, about $30 \mu \mathrm{g}$-sized meteorite samples were used. In order to evaluate the reproducibility, replicate analyses of the bulk Allende meteorite (Sample B) were carried out for different sizes (100 $\mu \mathrm{g}$ for B-1, $10 \mu \mathrm{g}$ for B-2).

\section{Results and Discussion}

\subsection{Isotopic analyses by direct-loading mass spectrometry}

Isotopic analyses were carried out using a double filament mode; Re-center and Ta-side filaments. Samples were loaded in nitride or chloride form and measured as metallic Li ion. Run currents under working conditions were $2.0 \mathrm{~A}$ (center) and $0.15 \sim$ $0.17 \mathrm{~A}$ (side) for analyses for spike and reagent standards, and $2.5 \mathrm{~A}$ (center) and $0.22 \sim$ $0.28 \mathrm{~A}$ (side) for direct-loading analyses of chondrules, bulk meteorites and standard rocks. To correct the discrimination caused by an electron multiplier, a square root of $6 / 7$ was multiplied to the observed ${ }^{6} \mathrm{Li} /{ }^{7} \mathrm{Li}$ ratio.

During the repeated runs, it was noted that the run current for a chloride form was generally lower than that for nitride form. The ${ }^{6} \mathrm{Li} /{ }^{7} \mathrm{Li}$ ratios obtained by using a chloride form were $0.0754 \pm 0.0004(1 \sigma)$, and systematically lower than those by using a nitride form (mean value: $0.07899 \pm 0.00009$ ). On the other hand, the ${ }^{6} \mathrm{Li} /{ }^{7} \mathrm{Li}$ ratios obtained by direct-loading technique for Allende bulk and chondrule samples were even higher than those of reagent standards, ranging from $0.0810 \pm 0.0009$ to $0.0828 \pm 0.0012$ (Table 1).

Compared to chemical reagent runs, direct-loading runs of Li require much higher heating temperature and yield more stable Li signals. Such features have been also 
Determination of Li Abundances in Chondrules, Bulk Chondrites, and Standard Rocks by Direct-Loading

Table 1. ${ }^{6} \mathrm{Li} /{ }^{7} \mathrm{Li}$ Ratios Determined by Direct-Loading Mass Spectrometry of Allende Bulk and Chondrule Samples, Compared with Those of Terrestrial Standards

\begin{tabular}{|c|c|c|c|}
\hline Run No. & Samples & Weight $^{3}$ & ${ }^{6} \mathrm{Li} /{ }^{7} \mathrm{Li}$ ratio ${ }^{6}$ \\
\hline Run 1 & Allende bulk & $100 \mu \mathrm{g}(\mathrm{Li}: 0.13 \mathrm{ng})$ & $0.0828 \pm 0.0012$ \\
\hline Run 2 & Allende bulk & $100 \mu \mathrm{g}(\mathrm{Li}: 0.13 \mathrm{ng})$ & $0.0810 \pm 0.0009$ \\
\hline Run 3 & Allende bulk & $100 \mu \mathrm{g}(\mathrm{Li}: 0.13 \mathrm{ng})$ & $0.0823 \pm 0.0013$ \\
\hline Run 4 & Allende $\mathrm{BO}^{\mathrm{c}}$ & $470 \mu \mathrm{g}(\mathrm{Li}: 0.94 \mathrm{ng})$ & $0.0816 \pm 0.0007$ \\
\hline \multirow[t]{2}{*}{ Run 5} & Allende $\mathrm{BO}$ & $370 \mu \mathrm{g}(\mathrm{Li}: 0.74 \mathrm{ng})$ & $0.0822 \pm 0.001 \overline{5}$ \\
\hline & & Weighted mean & $0.0818 \pm 0.0004$ \\
\hline \multicolumn{2}{|c|}{ Literature data: NBS ${ }^{11}$} & & $0.0828 \pm 0.0001$ \\
\hline \multicolumn{2}{|c|}{ NBS $^{(2)}$} & & $0.0820 \pm 0.0001$ \\
\hline \multicolumn{2}{|c|}{ IUPAC $(1989)^{10)}$} & & $0.0811 \pm 0.0023$ \\
\hline
\end{tabular}

a Weight of samples loaded on filaments.

b Errors are $1 \sigma$.

c BO: barred olivine chondrule.

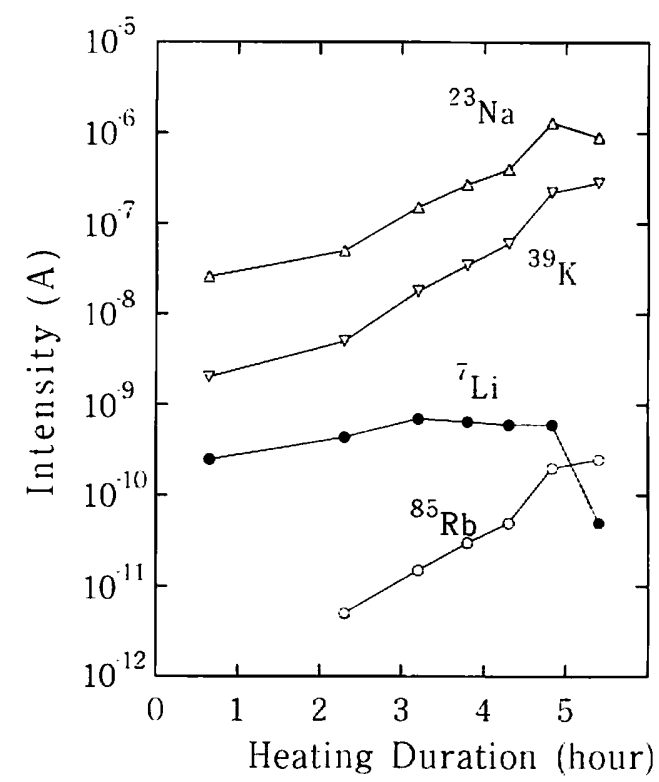

Fig. 1. The relative intensities of ${ }^{7} \mathrm{Li},{ }^{23} \mathrm{Na},{ }^{39} \mathrm{~K}$, and ${ }^{85} \mathrm{Rb}$ as a function of heating dura. tion, for $10 \mu \mathrm{g}$-sized BCR-1.

noted in cases of REE and other elements for direct-loading runs.9) As mentioned previously, in order to obtain a high efficiency of Li signals, matrix effects must be carefully controlled. Particularly, for high alkali samples, the influence of large amount of $\mathrm{Na}$ and $\mathrm{K}$ are significant on vaporization and ionization of $\mathrm{Li}$. For example, results for BCR-1 are shown in Fig. 1. It is pointed out that Na signal was always much $(2 \sim 3$ order magnitude) higher than that of $\mathrm{Li}$, and that $\mathrm{Na}$ (and $\mathrm{K}$ ) always interfered ionization of $\mathrm{Li}$. Therefore prolonged heating steps were required to minimize these interferences before heating up to reach to the best stable signals of Li. Otherwise, Li signals decay out quickly before $\mathrm{Na}$ and $\mathrm{K}$ decaying. Finally, the best Li signals can be obtained with reasonable heating schedule, which is similar to the cases for $\mathrm{Mg}, \mathrm{Ba}$, and $\mathrm{Sr}$ runs. ${ }^{91}$

It was found that the mass fractionation occurred more extensively during the 
Table 2. $\quad{ }^{6} \mathrm{Li} /{ }^{7} \mathrm{Li}$ Ratio for ${ }^{6} \mathrm{Li}$-Enriched Spike Solution

\begin{tabular}{cll}
\hline Run No. & \multicolumn{1}{c}{ Weight $^{3}$} & ${ }^{6} \mathrm{Li} /{ }^{7} \mathrm{Li}$ ratio \\
\hline Run 1 & Li: $1000(\mathrm{ng})$ & $21.54=0.20$ \\
Run 2 & Li: $1000(\mathrm{ng})$ & $21.66 \pm 0.30$ \\
Run 3 & Li: $1000(\mathrm{ng})$ & $21.64 \pm 0.21$ \\
Run 4 & Li: $1000(\mathrm{ng})$ & $21.50 \pm 0.21$ \\
& Weighted mean & $21.57 \pm 0.11$ \\
& Corrected & 22.35 \\
ORNL & & 22.15 \\
\hline
\end{tabular}

a Weight of samples loaded on samples.

b Errors are $1 \sigma$

c After corrected for mass fractionation.

Table 3. Li Concentration (in ppm) for ${ }^{6} \mathrm{Li}-\mathrm{E}$ nriched Spike Solution

\begin{tabular}{llll}
\hline Samples & $\mathrm{Sp} / \mathrm{Std}^{\mathrm{a}}$ & Weight $^{\mathrm{b}}$ & Li concentration $^{c}$ \\
\hline $\mathrm{S}(\mathrm{I})$ & $1.0 / 1.0$ & $\mathrm{Li}: 1000 \mathrm{ng}$ & $7.625 \pm 0.070$ \\
$\mathrm{~S}$ (I) & $1.0 / 1.0$ & $\mathrm{Li}: 500 \mathrm{ng}$ & $7.565 \pm 0.071$ \\
$\mathrm{~S}$ (I) & $1.0 / 1.0$ & $\mathrm{Li}: 500 \mathrm{ng}$ & $7.615 \pm 0.021$ \\
$\mathrm{~S}$ (II) & $1.0 / 0.5$ & $\mathrm{Li}: 200 \mathrm{ng}$ & $7.546 \pm 0.025$ \\
$\mathrm{~S}$ (II) & $1.0 / 0.5$ & $\mathrm{Li}: 100 \mathrm{ng}$ & $7.553 \pm 0.030$ \\
$\mathrm{~S}$ (III) & $0.5 / 1.0$ & Li: $100 \mathrm{ng}$ & $7.588 \pm 0.033$ \\
& & Weighted mean & $7.581 \pm 0.013$ \\
\hline
\end{tabular}

The proportion of the weight of spike solution to that of standard solution in the mixed solution.

beight of samples loaded on filaments.

c Errors are $1 \sigma$.

reagent run rather than the direct-loading run. For the direct-loading run, Li exists as minor component on a filament and may thus be stabilized by other major elements such as $\mathrm{Mg}, \mathrm{Ca}, \mathrm{Fe}$, etc. As shown in Table 1 , the mean value of ${ }^{6} \mathrm{Li} /{ }^{7} \mathrm{Li}$ ratio for Allende chondrules and bulk sample is $0.0818 \pm 0.0004$. This value is in good agreement with the recommended value of IUPAC $(1989)^{101}$ and also those of recent precise Li measurements. ${ }^{11 .}{ }^{121}$ The ${ }^{6} \mathrm{Li} /{ }^{i} \mathrm{Li}$ ratios in bulk meteorites are normally constant, ty pically 0.0813 as reported by Shima and Honda. ${ }^{13)}$

From the above discussion, the ${ }^{6} \mathrm{Li} /{ }^{7} \mathrm{Li}$ ratios obtained by direct-loading runs for Allende samples are considered to represent the least fractionated one and thus employed as a reference value for isotope dilution calculation in this work. The mean ${ }^{6} \mathrm{Li} /{ }^{7} \mathrm{Li}$ ratio obtained for nitride form is $0.07899 \pm 0.00009$. Then the fractionation factor of 0.966 is calculated relative to that of direct-loading value of Allende. The correction factor of 1.036 is therefore employed for correction of values obtained for reagent and spike solutions.

\subsection{Calibration of ${ }^{6} \mathrm{Li}$-enriched spike solution}

For concentration calibration of ${ }^{6} \mathrm{Li}$-enriched spike solution, the isotopic analyses of spike solution were, at first, carried out. The results of the ${ }^{6} \mathrm{Li} /{ }^{7} \mathrm{Li}$ ratios in nitride form are shown in Table 2. The mean value of $21.57 \pm 0.07$ obtained in this work is systematically lower than the ORNL value (22.15). Considering that this ratio was obtained in the reagent runs, a lower value may be caused by mass fractionation. Then 
Determination of Li Abundances in Chondrules, Bulk Chondrites, and Standard Rocks by Direct-Loading

Table 4. Comparison of Analytical Results of Lithium Abundances (in ppm) for Standard Rocks

\begin{tabular}{cccc}
\hline Sample & ${\text { Weight }(\mu \mathrm{g})^{\mathrm{i}}}^{\mathrm{i}}$ & This work $^{\mathrm{b}}$ & Literature $^{\mathrm{a}}$ \\
\hline JB-1 & 50 & $11.4 \pm 0.08$ & $11.5^{\mathrm{d}}$ \\
& 50 & $11.1 \pm 0.09^{\mathrm{c}}$ & \\
JB-2 & Weighted mean & $11.3 \pm 0.06$ & $8.0^{\mathrm{d}}$ \\
BCR-1 & 50 & $8.23 \pm 0.11$ & $13.5 \pm 1.7^{\mathrm{c}}$ \\
& 50 & $13.8 \pm 0.13$ & $12.9 \pm 0.4^{\mathrm{i}}$ \\
& 20 & $13.7 \pm 0.08^{\mathrm{c}}$ & \\
\hline
\end{tabular}

a Weight of samples loaded on filaments.

- Errors are $1 \sigma$.

'Replicate analyses for the same split solution.

¿ From Ando et al. ${ }^{14)}$

" From the mean value compiled in Gladney et al. ${ }^{151}$

' From the value obtained by isotope dilution method compiled in Gladney et al. ${ }^{15}$

Table 5. Results for Bulk Samples of Chondrites and Allende Chondrules

\begin{tabular}{|c|c|c|c|c|c|c|c|c|c|c|c|c|}
\hline Sample & Type $^{\bar{a}}$ & $\begin{array}{c}\text { Weight } \\
\text { (mg) }\end{array}$ & $\begin{array}{c}\text { Weight }^{\mathrm{c}} \\
(\mu \mathrm{g})\end{array}$ & $\begin{array}{c}\mathrm{Li} \\
(\mathrm{ppm})\end{array}$ & $\begin{array}{c}\mathrm{Na} \\
(\mathrm{ppm})\end{array}$ & $\begin{array}{c}\mathrm{K} \\
(\mathrm{ppm})\end{array}$ & $\begin{array}{c}\mathrm{Rb} \\
(\mathrm{ppm})\end{array}$ & $\begin{array}{c}\mathrm{Sr} \\
(\mathrm{ppm})\end{array}$ & $\begin{array}{c}\mathrm{Ba} \\
(\mathrm{ppm})\end{array}$ & $\begin{array}{l}\mathrm{Mg} \\
(\%)\end{array}$ & $\begin{array}{c}\mathrm{Ca} \\
(\%)\end{array}$ & $\begin{array}{l}\mathrm{Fe} \\
(\%)\end{array}$ \\
\hline \multicolumn{13}{|c|}{ Bulk meteorites } \\
\hline Allende $A^{d}$ & $\mathrm{CV}$ & 9.6 & 30 & 1.51 & n.a. & 335 & 1.19 & 19.8 & 6.36 & 12.4 & 1.81 & 11.1 \\
\hline B-1 & & 41.2 & 100 & 1.33 & n.a. & 301 & 1.19 & n.a. & n.a. & n.a. & n.a. & n.a. \\
\hline B- 2 & & 41.2 & 10 & 1.30 & n.a. & 303 & 1.21 & n.a. & n.a. & n.a. & n.a. & n.a. \\
\hline Ningqiang & $\mathrm{CK}$ & 16.4 & 30 & 1.78 & n.a. & 317 & 1.21 & 10.9 & 4.72 & 14.2 & 1.43 & 22.9 \\
\hline Murchison & $\mathrm{CM}$ & 11.1 & 30 & 1.65 & n.a. & 545 & 1.82 & 12.9 & 4.82 & 12.3 & 1.43 & 18.2 \\
\hline Allegan & H5 & 15.5 & 30 & 2.18 & n.a. & 867 & 2.04 & 11.3 & 4.25 & 17.0 & 1.34 & 15.1 \\
\hline Etter & L5 & 10.0 & 30 & 1.89 & n.a. & 650 & 2.04 & 11.8 & 4.16 & 11.6 & 1.07 & 16.6 \\
\hline \multicolumn{13}{|c|}{ Allende chondrules } \\
\hline SN-1 & $\mathrm{PO}$ & 6.95 & 30 & 1.34 & 2915 & 300 & 1.03 & 10.1 & 3.60 & 17.9 & 1.30 & 23.3 \\
\hline $\mathrm{SN}-2$ & POP & 7.57 & 30 & 1.87 & 7879 & 800 & 3.80 & 7.61 & 3.93 & 19.8 & 1.24 & 5.09 \\
\hline SN.7 & POP & 3.31 & 30 & 1.31 & 9433 & 604 & 1.99 & 18.5 & 5.03 & 14.8 & 1.83 & 8.45 \\
\hline SN-8 & POP & 2.15 & 30 & 1.82 & 7758 & 776 & 2.45 & 34.5 & 5.71 & 15.3 & 3.16 & 14.2 \\
\hline SN-14 & $\mathrm{BO}$ & 4.55 & 30 & 1.63 & 5397 & 462 & 1.74 & 11.4 & 5.30 & 18.6 & 1.31 & 19.8 \\
\hline $\mathrm{H}-1$ & POP & 10.5 & 30 & 2.38 & 5952 & 708 & 2.56 & 10.6 & 4.34 & 24.7 & 2.04 & 11.0 \\
\hline $\mathrm{H}-374$ & $\mathrm{PO}$ & 4.88 & 30 & 1.36 & 2796 & 305 & 1.03 & 9.81 & 3.68 & 19.2 & 1.14 & 23.1 \\
\hline
\end{tabular}

a PO: porphyritic olivine, POP: porphyritic olivine and pyroxene, BO: barred olivine.

b Weights of bulk chondrites decomposed or total weights of chondrules.

c Weights of samples loaded on filaments.

d Samples A and B were taken from a common powder split but decomposed separately. B-1 and B-2 are replicate analyses for the sample $B$.

e Not analyzed.

the correction factor of 1.036 was applied to the observed ${ }^{6} \mathrm{Li} /{ }^{7} \mathrm{Li}$ ratios of individual runs. The ${ }^{6} \mathrm{Li} /{ }^{7} \mathrm{Li}$ ratio of ${ }^{6} \mathrm{Li}$-enriched spike solution after the above correction is now in agreement with the value given from ORNL.

Calibration of the Li concentration in ${ }^{6} \mathrm{Li}$-enriched spike solution using mixed spike-standard solutions was then carried out. The results are shown in Table 3 . The Li concentrations determined for spike solutions were in agreement within $1 \%$. A mean 


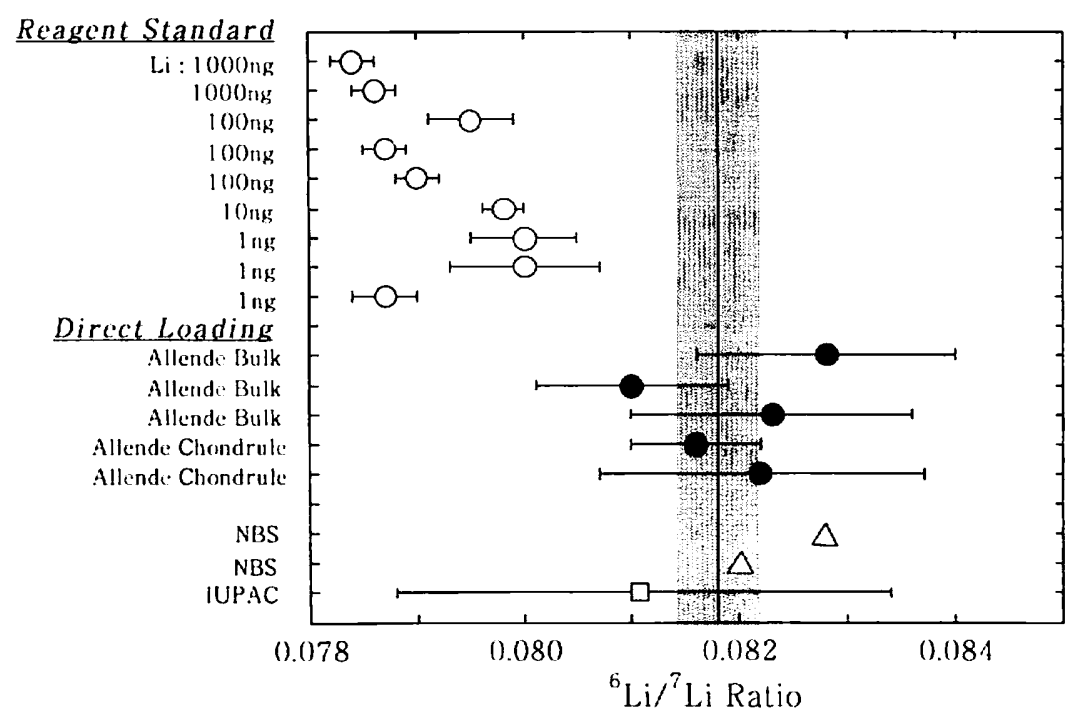

Fig. 2. Li isotopic compositions for reagent $\mathrm{Li}$ standard, Allende bulk, and chondrule samples compared with IUPAC $(1989)^{101}$ and NBS literature data. ${ }^{11 .}{ }^{12}$ Errors represent one standard deviation from the mean values. The dark area is one standard deviation error from the grand mean for Allende samples.

Li concentration of the stock solution ( $\mathrm{Li} 7.581 \pm 0.013 \mathrm{ppm}$ ) was used for later analyses of standard rocks, bulk meteorites, and chondrules.

3.3 Direct-loading analyses for standard rocks, bulk meteorites, and chondrules

Results for DL-IDMS of GSJ and USGS standard rocks (JB-1, JB-2 and BCR-1) and bulk meteorites are given in Tables 4 and 5 . In order to evaluate the reproducibility, replicate analyses of standard rocks (JB-1 and BCR-1) and the Allende meteorite were carried out. It is found that the results agree within $2 \sim 3 \%$ with each other. A slightly larger deviation in $\mathrm{Li}$ values was observed even for the same powder split of Allende, which may be due to the heterogeneous distribution of Li-enriched components such as CAIs." For standard rocks, our value for JB-2 is $3 \%$ higher than the literature value, but those for JB-1 are in good agreement with those given in Ando et al. ${ }^{14}$ On the other hand, our BCR-1 value is in good agreement with the mean value (13.5 \pm 1.7$)$ compiled in Gladney et $a{ }^{15}{ }^{(5)}$ but $7 \%$ higher than the mean value obtained by isotope dilution method

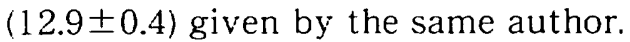

Although our results of standard rocks are a little dispersed from the literature data, no systematical differences were observed. Therefore the interlaboratory biases were not found. A total procedural blank of $\mathrm{Li}$ is $0.09 \mathrm{pg}$. The contribution of the Li blank to the sample was less than $0.01 \%$ and therefore was negligible. The contribution of other element blanks to the samples were less than $1 \%$. In view of the blank contribution, spike calibration and reproducibility, the precision of our analyses for standard rocks and meteorite samples is believed to be better than $3 \%$.

For meteorite samples, the abundances of other alkali metals ( $\mathrm{K}$ and $\mathrm{Rb}$ ), alkaline earths ( $\mathrm{Mg}, \mathrm{Ca}, \mathrm{Sr}$, and $\mathrm{Ba}$ ), and $\mathrm{Fe}$ were successively determined. Lithium abundances in bulk meteorites range from 1.30 to $2.18 \mathrm{ppm}$ and therefore are within the typical 
range of bulk chondrites. Except for a few case, most of other elements also show typical abundances for bulk chondrites. However, $\mathrm{Fe}$ abundances of Allende and Allegan and $\mathrm{Mg}$ and $\mathrm{Ca}$ abundances of Etter are too low for bulk chondrites. The reason of these low values is not understood. It may be possible that sampling heterogeneities exist and/or possible misleading mass spectrometric processes may not be ruled out.

Seven individual Allende chondrules were analyzed in this work. Li abundances range from 1.31 to $2.38 \mathrm{ppm}$ with a mean of $1.67 \mathrm{ppm}$. Previously some Li abundance data have been reported for chondrules from unequilibrated chondrites including Allende, ${ }^{5-7 !}$ but data for individual chondrules have not been presented. They range from 0.9 to $3.1 \mathrm{ppm}$ with one exception. Our mean value of Allende chondrules is consistent with their results, and is similar to the Allende whole rock values, as previously reported for several chondrites. ${ }^{51,71}$ Our data show that Li abundances in individual chondrule vary by less than a factor of 2 . It is thus pointed out that the $\mathrm{Li}$ concentrations are less variable in chondrules, which is in contrast with the large variations for other alkali metal and alkaline earth elements.

In this work, only seven chondrules were analyzed for Li. To establish more general abundance feature for chondrules, further systematic analytical works would be required in future.

\section{Acknowledgments}

We are indebted to Dr. B. Mason for providing us with specimens of the Allegan meteorite, and to Dr. E. J. Olsen for the Murchison meteorite. We are also indebted to Dr. W. Daode for the Ningqiang meteorite. We thank two anonymous reviewers for their constructive comments on an early version of the manuscript. This research was partly supported by a Grant-in-Aid for General Scientific Research from the Ministry of Education, Science, and Culture of Japan.

\section{References}

1) E. M. Burbidge, G. R. Burbidge, W. A. Fowler, and F. Hoyle, Rev. Mod. Phys., 29, 547 (1957).

2) M. Quijano-Rico and H. Wänke, "Meteorite Research," ed. by P. Millman, D. Reidel, Dordrecht (1969), p. 132.

3) W. Nichiporuk and C. B. Moore, Earth Planet. Sci. Lett., 9, 280 (1970).

4) W. Nichiporuk and C. B. Moore, Geochim. Cosmochim. Acta, 38, 1691 (1974).

5) S. V.S. Murty, P. N. Shukla, and P. S. Goel, Meteoritics, 18, 123 (1983).

6) D. Krankowsky and O. Müller, Geochim. Cosmochim. Acta, 31,1833 (1967).

7) H. Balsiger, J. Geiss, N. Groegler, and A. Wyttenbach, Earth Planet. Sci. Lett., 5,17 (1968).

8) G. Dreibus, B. Spettel, and H. Wänke, Proc. Lunar Planet. Sci. Conf. 7 th (1976), p. 3383.

9) N. Nakamura, K. Yamamoto, S. Noda, Y. Nishikawa, H. Komi, H. Nagamoto, T. Nakayama, and K. Misawa, Analyt. Chem., 61, 755 (1989).

10) IUPAC CAIWA SIA.M, Pure Appl. Chem., 63, 991 (1991).

11) L. H. Chan, Analyt. Chem., 59, 2662 (1987).

12) L. W. Green, J. J. Leppinen, and N. L. Elliot, Analyt. Chem., 60, 34 (1988).

13) M. Shima and M. Honda, Geochem. J., 1, 27 (1966).

14) A. Ando, N. Mita, and S. Terashima, Geostand. Newsl., 11, 159 (1987).

15) E. S. Gladney, C. E. Burns, and I. Roelandts, Geostand. Neusl., 7, 3 (1983). 
N. Morikawa, N. Nakamura, S. Nakanishi, K. Yamamoto, and Y. Tanami

\section{Keywords}

Li

Direct-loading

Isotope dilution

Chondrule

Standard rock 